Please refer to this paper as:

Haslam, M., Arcelus, J., Farrow, C., \& Meyer, C. (2012). Attitudes towards emotional expression mediate the relationship between childhood invalidation and adult eating concern. European eating disorders review, 20(6), 510-514.

\title{
Attitudes towards emotional expression mediate the relationship between childhood invalidation and adult eating concern
}

\author{
Michelle Haslam, BSc ${ }^{a}$ \\ Jon Arcelus ${ }^{a},{ }^{b}$ \\ Claire Farrow ${ }^{\mathrm{a}}$ \\ Caroline Meyer, $\mathrm{PhD}^{\mathrm{a}}$
}

\begin{abstract}
aLoughborough University Centre for Research into Eating Disorders (LUCRED), School of Sport, Exercise and Health Science, Loughborough, LE11 3TU, UK

'Leicester Eating Disorder Service, Brandon Mental Health Unit, Leicester General Hospital, Gwendolen Rd, Leicester, Leicestershire LE5 4PW
\end{abstract}

\begin{abstract}
Previous research has suggested that invalidating childhood environments are positively related to the symptoms of eating disorders. However it is unclear how childhood environments might impact upon the development of eating disorder symptoms. This study examined the relationship between parental invalidation and eating disorder related attitudes in a nonclinical sample, and tested the mediating effect of attitudes towards emotional expression. Two hundred women, with a mean age of 21 , completed measures of invalidating childhood environments, attitudes towards emotional expression and eating pathology. Eating concerns were positively associated with recollections of
\end{abstract}


an invalidating parental environment. The belief that the expression of emotions is a sign of weakness fully mediated the relationship between childhood maternal invalidation and adult eating concern. Following replication and extension to a clinical sample, these results suggest that targeting the individual's attitude towards emotional expression might reduce eating attitudes among women who have experienced an invalidating childhood environment.

Key words: Invalidating childhood environment, attitude towards emotional expression, anorexia, bulimia

\section{Attitudes towards emotional expression mediate the relationship between childhood invalidation and adult eating concern}

\section{Introduction}

Childhood environments are linked to eating disordered attitudes and behaviours in later life (Schmidt, Humfress \& Treasure, 1998; Kent \& Waller, 2000). Research has focused on the child's development of emotional states and in particular the family's input to the child's processing of negative emotions (e.g. Kent \& Waller, 2000; Parker, 1983). In some cases, the child develops an understanding that negative emotional states are not acceptable and they respond by using 'blocking' mechanisms to reduce awareness of such states (Root \& Fallon, 1989; Waller, Kennerley 
\& Ohanian, 2007). These blocking mechanisms can include symptoms of eating disorders such as bingeing and purging, dietary restriction and compulsive exercise.

Previous research has also linked individuals' experience of negative emotions with childhood abuse and neglect (Kent \& Waller, 2000) and poor experiences of parental care (Parker, 1983). However, such research has not explained how the child develops the belief that negative emotions are unacceptable. Linehan (1993) coined the term 'invalidating childhood environment' which she defined as an environment where the child's emotional experiences are not validated by the parents and the expression of such emotions is met with punishment or neglect. Invalidating childhood environments have been found to be associated with eating disorders (Schmidt et al., 1989). In women with eating disorders, perceptions of an invalidating childhood environment have been related to negative core beliefs which are believed to perpetuate and maintain eating psychopathology (Ford, Waller \& Mountford, 2011). Mountford, Corstophine, Tomlinson \& Waller (2007) found that emotional invalidation was associated with poor distress tolerance, which in turn was related to eating disorder symptoms. In addition, Haslam, Mountford, Meyer and Waller (2008) concluded that such environments were more common in people who present with bulimic symptoms than other diagnostic groups. Specifically, vomiting was associated with paternal invalidation while the experience of an emotionally controlled, high achieving family style was associated with excessive exercise. In contrast, those who reported engagement in binge-eating were less likely to have had an invalidating relationship with their mother than those who were not binge-eating.

While evidence implicates parental invalidation in eating disorder psychopathology, it is unclear how an invalidating childhood environment could result in a higher chance of developing eating problems later in life. One possible mechanism is through the development of negative attitudes towards emotional expression. Previous research suggests that individuals with eating disorders experience, cope with, and express emotions differently to controls (e.g. Meyer, Waller \& Waters, 1998; Whiteside et al., 2007; Davies, Swan, Schmidt, \& Tchanturia, 2011; Wildes, Marcus, Bright, \& 
Dapelo \& Psychol, 2012). Negative attitudes towards emotional expression have previously been described by Meyer, Leung, Barry \& DeFeo (2010) who provide examples of such attitudes such as "I think getting emotional is a sign of weakness," "you should always keep your feelings to yourself," and "my bad feelings will harm others if I express them." In a non-clinical sample, Meyer, Leung, Barry \& DeFeo (2009) reported that those women who had high levels of eating, shape, and weight concern believed that displaying emotions is a sign of weakness. Those with high levels of weight concern were also likely to believe that they should keep their emotions under control and that others might reject them if they displayed emotions. In other research with clinical samples, women with anorexia nervosa have been found to inhibit the expression of emotions such as anger and sadness during interpersonal situations and often describe engaging with unhealthy eating related behaviours to manage such emotions (Espeset, Gulliksen, Nordbø, Skårderud, Holte, 2012). However, it is unclear how these negative attitudes towards emotional expression originate. It could be possible that such attitudes develop as a result of an invalidating childhood environment, where emotions are invalidated and rejected by caregivers. It is well documented that eating disordered symptoms are likely to manifest when an individual is experiencing negative emotional states (Grilo, Shiffman \& Carter-Campbell, 1998; Vanderlinden et al., 2001; Waters, Hill \& Waller, 2001), and the inability to express or cope with such states can lead to (or exacerbate) symptoms (Stickney, Miltenberger \& Wolff, 1999; Corstophine, 2006).

This study aims to replicate and extend the findings of Haslam et al., (2008) and Meyer et al., (2009) by exploring whether negative attitudes towards emotional expression mediate the relationship between invalidating childhood environments and eating disorder symptoms in adulthood. For the present study eating disorder symptoms are defined using the Eating Disorder Examination Questionnaire and are classified as restraint, eating concern, body shape concern, and body weight concern (Fairburn \& Beglin, 1994). It was hypothesised that eating disorder symptoms would be positively related to invalidating childhood environments, and that eating disorder symptoms would be positively related to negative and dysfunctional attitudes towards emotional expression. It was also hypothesised that attitudes towards emotional expression would 
significantly mediate the effect of invalidating childhood environments upon eating disorder symptoms in adulthood.

\section{Materials and Methods}

\section{Participant characteristics}

Two hundred female University students participated in this research (mean age $=21$ years; SD 3.7, range 18 - 54). Students reported their height and weight and their mean BMI $=21.7$ (SD 2.93 , range $15-36)$. Participants completed the following measures:

\section{The Eating Disorder Examination Questionnaire (EDE-Q; Fairburn \& Beglin, 1994)}

The EDE-Q measures restraint, eating concern, body shape concern, and body weight concern currently, focusing on the last 4 weeks. It also measures behaviours such as binge eating and selfinduced vomiting. High scores indicate more pathological eating attitudes and behaviours. The $E D E-Q$ is an effective screening tool for detecting eating disorders in various clinical populations (Black \& Wilson, 1996; Fairburn \& Beglin, 1994) and the measure has been used extensively in non-clinical populations (e.g. Haslam et al., 2008, Meyer, Leung, Barry \& DeFeo, 2010).

\section{The Attitudes towards Emotional Expression Scale (AEE; Joseph, Williams, Irwing, \& Cammock,} $\underline{1994)}$

The AEE measures the belief that the expression of emotions is a sign of weakness (weakness); the belief that emotions should be kept under control (control); the belief that other people will reject expressed emotions (social); and the tendency to keep emotions to oneself (non-expression). It is reliable and valid (Laghai \& Joseph, 2000) and has been used in clinical (Meyer, Leung, Barry \& De Feo, 2009) and non-clinical groups (Laghai \& Joseph, 2000).

The Invalidating Childhood Environment Scale (ICES; Mountford et al., 2007)

The ICES measures maternal and paternal behaviours, where participants are given statements about their parents (e.g. My parents would become angry if I disagreed with them). Participants are 
then asked to rate their experience with each parent up to the age of 18 years. Higher scores represent higher levels of perceived parental invalidation. The subscales have good internal consistency (Mountford et al., 2007). Four final items assess broad pictures of family style during childhood, which were proposed by Linehan (1993). Three of these are styles of invalidating environment ('typical', 'perfect', 'chaotic', and one description of an emotionally supportive environment ('validating').

\section{Data analysis}

Scores on the EDE-Q and AEE were not normally distributed, therefore nonparametric analyses were performed where possible. All tests were 1-tailed, in keeping with the directional hypotheses. In order to assess the $1^{\text {st }}$ and $2^{\text {nd }}$ hypotheses, Spearman's rank correlations were used to explore the relationships between ICES, AEE and EDE-Q subscale scores. To assess the third hypothesis mediation analysis was used (Baron \& Kenny, 1986) to evaluate whether attitudes towards emotional expression mediated the relationships between invalidating childhood environment and dysfunctional eating attitudes and behaviours. Regression analyses were used to test for mediation as there is no non-parametric equivalent for regression. According to Allison (1999), normal distribution is the least important assumption of regression; instead emphasising other assumptions such as linearity and homoscedasticity of residuals and absence of multicollinearity, extreme outliers and leverage of residuals. These particular assumptions were met by the data and consequently regression analysis was deemed acceptable. According to Baron and Kenny (1986), mediation is evident if: (1) the independent variable predicts the dependent variable, (2) the independent variable predicts the mediator, (3) the mediator predicts the dependent variable, and (4) the independent variable no longer significantly predicts the dependent variable when entered into a regression with the mediator. Mediation analyses were performed where aspects of childhood invalidation, attitudes towards emotional expression and eating disorder symptoms were correlated.

\section{Results}




\section{Descriptive statistics for measures obtained}

Table 1 presents the sample characteristics for the measures obtained. Mean and standard deviation scores are comparable with other non-clinical available data (Mond, Hay, Rodgers, Owen \& Beumont, 2004; Meyer, Leung, Barry, \& De Feo, 2009).

Correlations between invalidating childhood environments, attitudes towards emotional expression and eating disorder symptoms

Table 2 shows the results of the correlations between the ICES, AEE and EDE-Q subscales. In keeping with the hypotheses, there was a significant, positive association between eating concern and the experience of maternal and paternal invalidation, and all AEE subscales. In addition, there was a significant, positive relationship between restraint and non-expression. Weight concern was positively correlated with paternal invalidation and all AEE subscales. There was a significant, positive relationship between shape concern and all AEE subscales. Associations between parental invalidation and EDE-Q subscales were different to the results of Haslam et al, (2008), as no correlations were found in their study, which used a clinical population. Associations between AEE and EDE-Q subscales are similar to those of Meyer at al., (2009), however non-expression was not correlated with EDE-Q subscales in their study. [Table 2 about here]

Attitude towards emotional expression as a mediator between invalidating childhood environments and eating concern

Mediation analyses tested whether attitudes towards emotional expression mediated the relationship between invalidating childhood environment with eating and weight concern as these variables were significantly related in the correlations described above. The hypothesised mediation models were not significant with weight concern as the dependent variable, or with nonexpression, control or social rejection as mediators. However, as indicated in Table 3, believing that expressing emotions are a sign of weakness fully mediated the relationship between maternal invalidation and eating concern. Viewing emotional expression as a sign of weakness failed to 
mediate the relationship between paternal invalidation and eating concern; Sobel test: $p>.05$ [Table 3 about here].

\section{Discussion}

The current study aimed to examine the link between invalidating childhood environments and eating disorder related attitudes and to determine whether these links are mediated by attitudes towards emotional expression. Results replicate findings that an invalidating childhood environment is associated with adult eating disorder symptoms and cognitions (Ford et al., 2011; Haslam et al., 2008). However while Haslam et al, (2008) found that invalidating childhood environments were not associated with eating disorder related attitudes in a clinical population, the present study found that positive associations were present in a non-clinical population. In addition, this study replicates previous findings that a negative attitude towards emotional expression is associated with eating concern (Espeset et al., 2012; Meyer et al., 2009). The current results extend previous findings by indicating that a negative attitude towards emotional expression fully mediates the relationship between maternal childhood invalidation and adult eating concern.

Several theories exist suggesting that eating disorder symptoms are used by patients in order to regulate emotional affect (e.g. Fox \& Power, 2009; Schmidt \& Treasure, 2006), in addition to the theoretical suggestion that childhood experiences play an aetiological role in the development of eating distress (e.g., O'Shaughnessy \& Dallos, 2009). The findings of this research add to this body of knowledge by beginning to provide evidence for how invalidating experiences during childhood may impact upon the development of eating disorder symptoms through shaping dysfunctional attitudes towards emotional expression. The significant mediation model reported here support a potential cognitive model where individuals who experienced an invalidating maternal relationship are more likely to view emotional expression as a sign of weakness. When a child's emotions are ignored or met with punishment by the mother, the child potentially learns to view their emotions as incorrect, and learns to control them in order to avoid punishment or rejection. As a result, they may 
be less able to label and cope with their negative emotions. This in turn, could lead to or exacerbate existing eating disorder related attitudes, specifically eating concern. As eating disordered behaviours are likely to manifest when an individual is experiencing negative emotional states (Grilo, Shiffman \& Carter - Campbell, 1998; Vanderlinden et al., 2001; Waters, Hill \& Waller, 2001), the inability to express or cope with such states can lead to or exacerbate symptoms (Corstophine, 2006).

This study has explored a potentially useful model. The focus of the present study is a nonclinical sample; it is well documented that women with eating disorders have difficulty in reporting their experiences accurately (e.g., Swan \& Andrews, 2003) and this is particularly relevant for studies employing the ICES, as the measure requires individuals to recall experiences from their childhood. Future research requires a longitudinal design to clarify the causality in any such relationships to explore clinically relevant symptoms. In addition, the cross sectional and retrospective design of the current study means that the study cannot address causality. It also remains unclear why other aspects of negative attitude towards emotional expression did not mediate the relationship between maternal invalidation and eating concern and this study may be underpowered to detect all significant relationships. For example, while viewing emotional expression as a sign of weakness mediated this relationship, the belief that emotions should be controlled, the belief that if you express emotions others will reject you, and the behaviour of not expressing emotions, did not play a mediating role. Future research could explore these differences, as well as addressing why maternal invalidation was a mediator but paternal invalidation was not, possibly exploring whether mothers' validation plays a larger role as mothers are usually the primary caregiver.

The results of the current study suggest that addressing attitude towards emotional expression among individuals with eating disorder related attitudes could provide a potential way of enhancing treatment efficacy. However associations require replication in a clinical group before firm conclusions can be drawn regarding implications for treatment. Previous research has concluded 
that challenging negative attitudes towards emotional expression could help facilitate the acceptance of such emotions and enable the individual to learn more healthy emotion regulation strategies (Waller, Corstophine, \& Mountford, 2007). On a practical level clinicians may find it useful to explore not only emotional acceptance and develop effective strategies to help patients cope with negative emotions, but addressing the underlying factors that may precede negative attitudes towards emotional expression may help to develop effective treatments for those with disordered attitudes towards eating.

\section{References}

Baron, R, M., \& Kenny, D. A. (1986). The moderator-mediator variable distinction in social psychological research: Conceptual, strategic, and statistical considerations. J Pers Soc

Psychol, 51, 1173-1182.

Black, C. M., \& Wilson, G. T. (1996). Assessment of eating disorders: interview versus questionnaire. International Journal of Eating Disorders, 20, 43-50.

Corstorphine, E. (2006). Cognitive-emotional-behavioural-therapy for the eating disorders: Working with beliefs about emotions. Eur Eat Disord Rev, 14, 448-461. 
Davies, H., Swan, N., Schmidt, U., \& Tchanturia, K. (2011). An Experimental Investigation of Verbal Expression of Emotion in Anorexia and Bulimia Nervosa, Eur Eat Disord Rev, 22. doi:

10.1002/erv.1157

Espeset, E.M., Gulliksen, K.S., Nordbø, R.H., Skårderud, F., \& Holte, A. (2012). The Link Between Negative Emotions and Eating Disorder Behaviour in Patients with Anorexia Nervosa, Eur Eat Disord Rev, 14. doi: 10.1002/erv.2183.

Fairburn, C. G., \& Beglin, S. J. (1994). Assessment of eating disorders: Interview or self report. International Journal of Eating Disorders, 16, 363-370.

Ford, G., Waller, G., \& Mountford, V. (2011). Invalidating childhood environments and core beliefs in women with eating disorders, Eur Eat Disord Rev, 19(4): 316-21. doi: 10.1002/erv.1053.

Fox, J. R., \& Power, M. J. (2009). Eating disorders and multi-level models of emotion: An integrated model. Clinical Psychology \& Psychotherapy, 16, 240-267.

Grilo, C. M., Shiffman, S., \& Carter-Campbell, J. T. (1994). Binge eating antecedents in normal weight nonpurging females: Is there consistency? International Journal of Eating Disorders, 16, 239-249.

Haslam, M., Mountford, V., Meyer, C., \& Waller, G. (2008). Invalidating childhood environment in anorexia and bulimia nervosa. Eating Behaviors, 9, 313-318.

Joseph, S., Williams, R., Irwing, P., \& Cammock, T. (1994). The preliminary development of a measure to assess attitudes towards emotional expression. Personality and Individual Differences, 16, 869-875.

Kent, A., \& Waller, G. (2000). Childhood emotional abuse and eating pathology. Clinical Psychology Review, 20, 887-903.

Laghai, A., \& Joseph, S. (2000). Attitudes towards emotional expression: Factor structure, convergent validity and associations with personality. British Journal of Medical Psychology, 73, 381-384.

Linehan, M. M. (1993). Cognitive-behavioral treatment of borderline personality disorders. Guilford: New York, NY. 
Meyer, C., Leung, N., Barry, L., \& De Feo, D. (2010). Emotion and Eating

Psychopathology: Links with Attitudes Toward Emotional Expression Among Young

Women. International Journal of Eating Disorders, 43 (2) 187-189.

Meyer, C., Waller, G., Waters, A. (1998). Emotional states and bulimic psychopathology. In: Hoek $\mathrm{H}$, Treasure, \& J, Katzman (eds). The Integration of Neurobiology in the Treatment of Eating Disorders. Chichester: Wiley, pp. 271-289.

Mountford, V., Corstorphine, E., Tomlinson, S., \& Waller, G. (2007). Development of a measure to assess invalidating childhood environments in the eating disorders. Eating Behaviors, 8, 48-58.

O'Shaughnessy R, \& Dallos R. (2009). Attachment research and eating disorders: a review of the literature, Clin Child Psychol Psychiatry, 14(4): 559-74.

Parker, G. (1983). Parental overprotection: A risk factor in psychosocial development. New York: Grune \& Stratton.

Root, M. P. P., \& Fallon, P. (1989). Treating the victimized bulimic. Journal of Interpersonal Violence, 1, 90-100.

Schmidt U, Humfress H, \& Treasure J. (1998). The role of general family environment and sexual and physical abuse in the origins of eating disorders. European Eating Disorders Review, 5, 184-207.

Schmidt, U., \& Treasure, J. (2006). Anorexia nervosa: Valued and visible. A cognitive-interpersonal maintenance model and its implications for research and practice. British Journal of Clinical Psychology, 45, 343-366.

Swan, S., \& Andrews, B. (2003). The relationship between shame, eating disorders and disclosure in treatment. British Journal of Clinical Psychology, 42, 367-378.

Vanderlinden, J., Dalle-Grave, R., Vandereycken, W., Noorduin, C. (2001). Which factors do provoke binge eating? An exploratory study in female students. Eating Behaviors, 2, 79-83.

Waller, G., Corstorphine, E., \& Mountford, V. (2007). The role of emotional abuse in the eating disorders: Implications for treatment. Eat Disord, 15, 317-331. 
Waller, G., Kennerley, H., \& Ohanian, V. (2007). Schema-focused cognitive behavioral therapy with eating disorders. In L. P. Riso, P. L. du Toit, D. J. Stein, \& J. E. Young (Eds.), Cognitive schemas and core beliefs in psychiatric disorders: A scientist-practitioner guide (pp. 139-175). New York: American Psychological Association.

Waters, A., Hill, A., \& Waller, G. (2001). Internal and external antecedents of binge eating episodes in a group of women with bulimia nervosa. Int J Eat Disord, 29, 17-22.

Whiteside, U., Chen, E., Neighbors, C., Hunter, D., Lo, T., Larimer, M. (2007). Difficulties regulating emotions: Do binge eaters have fewer strategies to modulate and tolerate negative affect? Eat Behav, 8, 162-169.

Wildes, J.E., Marcus, M.D., Bright, A.C., Dapelo, M.M., \& Psychol, M.C. (2012). Emotion and eating disorder symptoms in patients with anorexia nervosa: An experimental study, Int J Eat Disord, 31. doi: 10.1002/eat.22020.

Table 1: Descriptive statistics for the measures obtained.

\begin{tabular}{cll}
\hline & Mean & SD \\
\hline EDE-Q Restraint & 1.54 & 1.49 \\
Weight concern & 2.22 & 1.57 \\
Eating concern & 0.9 & 1.08 \\
Shape concern & 2.31 & 1.57 \\
\hline AEE Sign of weakness & 10.23 & 3.27 \\
Non-expression & 11.70 & 3.35 \\
Control & 12.19 & 3.53 \\
\hline
\end{tabular}




\begin{tabular}{ccc}
\hline Social rejection & 12.82 & 2.69 \\
\hline ICES: Maternal invalidation & 2.33 & 0.26 \\
Paternal invalidation & 2.40 & 0.34 \\
Chaotic & 1.41 & 1.12 \\
Validating & 4.21 & 1.12
\end{tabular}

ICES=invalidating childhood environment scale; AEE=attitudes towards emotional expression; EDEQ=eating disorder examination questionnaire

Table 2: Spearman's 1-tailed correlations between ICES, AEE and EDEQ subscales

\begin{tabular}{|c|c|c|c|c|}
\hline ICES scales & Restraint & $\begin{array}{l}\text { Eating } \\
\text { concern }\end{array}$ & $\begin{array}{l}\text { Weight } \\
\text { concern }\end{array}$ & $\begin{array}{l}\text { Shape } \\
\text { concern }\end{array}$ \\
\hline Maternal invalidation & -.026 & $.128^{*}$ & .061 & .054 \\
\hline Paternal invalidation & .112 & $.169^{* *}$ & $.177^{* *}$ & .107 \\
\hline \multicolumn{5}{|l|}{ AEE scales } \\
\hline Sign of weakness & .075 & $.272^{* *}$ & $.247^{* *}$ & $.236^{* *}$ \\
\hline Non expression & $.212^{* *}$ & $.275^{* *}$ & $.298^{* *}$ & $.227^{* *}$ \\
\hline
\end{tabular}


Control

Social rejection
.089

.016
$.171^{* *}$

$.156^{*}$
$.179^{* *}$

$.142^{*}$ $.188^{\star *}$

$.156^{*}$

${ }^{*} \mathrm{p}<.05 * * \mathrm{p}<.01$ ICES=invalidating childhood environment scale; AEE=attitudes towards emotional expression; EDEQ=eating disorder examination questionnaire

Table 3: Unstandardised beta coefficients $(b)$ for the mediation models

\begin{tabular}{|c|c|c|}
\hline Independent variable & Maternal invalidation & Paternal invalidation \\
\hline Mediator & Sign of & Sign of \\
\hline & weakness & weakness \\
\hline Dependent variable & Eating concern & Eating concern \\
\hline Step 1: Regression using parental invalidation to predict & $0.17^{*}$ & $0.20^{* *}$ \\
\hline
\end{tabular}


Step 2: Regression using parental invalidation to predict

the attitudes towards emotional expression

Step 3: Regression using attitudes towards emotional

expression to predict eating concern

Step 4: Regression using parental invalidation and

attitudes towards emotional expression to predict eating

concern

Parental invalidation

Attitudes towards emotional expression
0.14

$0.17^{* a}$

$0.14^{*}$

$0.14^{*}$

$0.23^{* *}$

$0.23^{* *}$

$0.14^{* *}$

$0.20^{* *}$

\footnotetext{
${ }^{*} p<0.05{ }^{* *} p<0.01$ a Sobel test indicated that partial mediation was not significant
} 